\title{
CHRONOMCS AND CHRONOBIOLOGY IN HEALTH AND DISEASE
}

\author{
R K Singh*
}

\author{
Biochemistry Department, Chhatrapati Shahuji Maharaj Medical University ( Formerly King George's Medical University, \\ Lucknow- 226003, India.
}

Chronobiology is a branch of science that objectively explores and quantifies mechanisms of biological time structure including important rhythmic manifestations of life right from molecular level of living being, from unicellular organism to complex organism such as human being. Genetics eventually leads to genomics, concurrently the study of biological variability i.e. chronobiology leads to chronomics.

GENETICS, the study of biological diversity in space CHRONOBIOLOGY, the study of biological diversity in time GENOMICS, the mapping of biological diversity in space i.e. of genomes in us

CHRONOMICS, the mapping of biological diversity in time, i.e. of chronomes (from chronos, time and monos, rule ) time structure of each pertinent variable in and around us, near and far.

Chronobiology, the study of mechanisms underlying diversity in time and chronomics, the mapping of chronomes - time structures, could complement genetics. It is the study of mechanisms underlying diversity in space and also genomics, the mapping of genomes. Chronobiology allows us to approach risks, diagnosis and treatment dependent on appointment time, especially of the dynamics of time, gender, age, ethnicity and geographical location. Chronobiologic reality consists of a multifrequency spectrum of rhythms. Homeostasis, probably, needs to be replaced by an approach such as chronobiology that considers predictable variability within the physiologic range. Chronobiology hence renders predictable and exploits what otherwise is considered as exclusively random variation. Chronomics is chronophysiological genomics, not based upon the concept of the genes underlying the true blood pressure

\section{Address for Correspondence :}

\section{Prof. R K Singh}

Biochemistry Department,

Chhatrapati Shahuji Maharaj Medical University (Formerly King George's Medical University ), Lucknow-226003.

E-mail:singhrk23a@hotmail.com; raj_singh509@yahoo.com or the true heart rate, but replacing the historically useful homeostatic truisms by the dynamics end points of a variable in the biosphere and/or in its cosmos (Fig 1). Apart from providing a better understanding of the universe, common to both nuclear physics and chronobiology, perhaps one of the most challenging applications of chronobiology is that of improving the quality of health care of individuals, societies and environments while so reducing its cost by focusing on detecting and treating risk to prevent overt diseases again of societies, such as homicide and war, and of individuals, such as severe vascular disease or cancer. For such aims, chronomics offers the opportunity to refine the definition and assessment of health and broader normalicy positively and individually, rather than quantifying disease as a deviation from normalcy, negatively as the absence of disease and only for populations, as percent morbidity and mortality. Early time structure alternation indicates earlier risk elevation. Herein, we try to note the cost-effectiveness of using a database of vascular chronomes for severe disease prevention. Chronomics specifically resolves risks of stroke and other severe incapacitating diseases, higher than the risk associated with hypertension and other oncological and psychiatric disease risks.

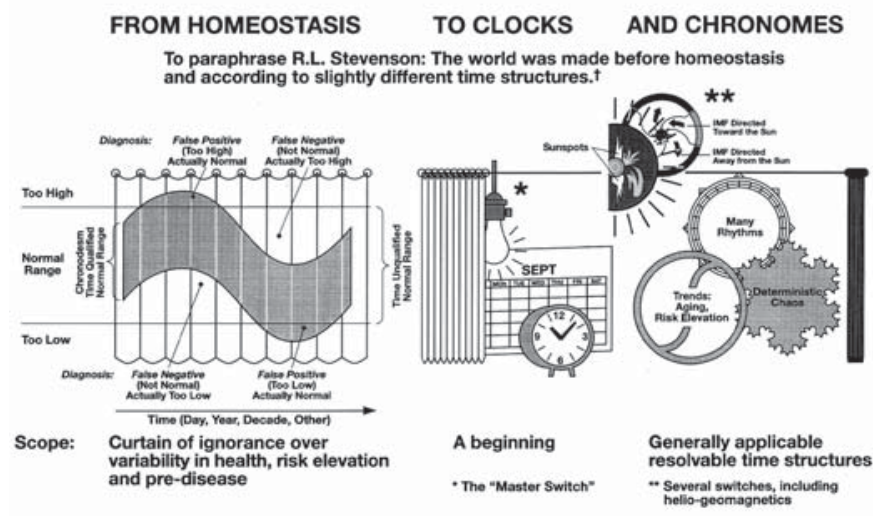

Fig 1: Variation within the physiological range can be resolved into meaningful information, including rhythms with frequencies covering 10 orders of magnitude, far beyond the circadian system and seasonal changes. Non-photic as well as photic influences of the sun have been genetically preserved in a set of photo- and magneto- periodicities from which much can be learned. (c) Halberg 
The rhythmic structure of living organisms is partly endogenous, genetically programmed and thus differentially and rhythmically responsive to environmental cycles. From the medical viewpoint, a deviation from the physiologic range serves mainly to diagnose overt disease. Chronobiologic considerations, in turn, aim at chronorisk assessment by values that may lie well within the physiological range as a step toward prevention (1). Body rhythms have been shown to affect not only daily, weekly, monthly and yearly functions, but also the prevalence of disease symptoms, medical test results and even the way the body responds to drug therapies.

Ecclesiastes 3: 1-8 insists that for everything there is a season and a time for every matter under heaven. A time to be born and a time to die; a time to heal and a time to breakdown, and a time to build up; a time to plant and a time to pluck up what is planted. God has made everything beautiful in its time. Present day chronobiologists began discovering conditions for which there would indeed be a best time to give medications, to make organ transplants, for malnourished to eat, for surgeons to operate and so on. The occurrence of disease is not a random process; rather there is a biological time structure to it. Timing can be manipulated in much the same way as dosing. Chronobiology offers new concepts that can be applied for making recommendations as to when to sample and when to treat. Some of these concepts can be incorporated into today's clinical practice without the need to make major adjustments to the actual clinical procedures in use such as the timed use of corticosteroids, oncostatics, cyclosporine or antihypertensives and a long list of other drugs (2-5).

Professor Franz Halberg has dedicated almost 60 years of his active and productive life to chronobiological research; who is known as the Father of Chronobiology. His long lasting basic scientific work is directed, at the beginning of the new century, to chronobiometry ( physiological and statistical evaluation of the genetically anchored and cosmically influenced time structures), chronobioengineering ( collecting physiological data by means of sophisticated equipments ), chronobiological diagnosis of disease risk syndromes the chronotherapy, improvement of prognosis, treatment in different fields of medicine and last but not the least, chronoastrobiology focusing on rhythms and broader chronomes to explore the origins of life $(6,7)$. It has been understood for centuries that the movements of the earth in relation to the sun produce seasonal and daily cycles in light energy and that these have had profound effects on the evolution of life. It is now emerging that rhythmic events generated from within the sun itself, as a large turbulent magnet in its own right, can have direct effects upon life on earth. Moreover, comparative studies of diverse species indicate that there have also been ancient evolutionary effects shaping the endogenous chronomic physiological characteristics of life. Thus the rhythms of the sun can affect us not only directly, but also indirectly through the chronomic patterns that solar magnetic rhythms have created within our physiology in the remote past. According to Prof Halberg, biological rhythms can redefine diagnosis and therapy (3). We feel deeply honored to have had the support, cooperation and supervision of Prof. Halberg since 1977 in carrying out our chronobiological research. We published our first paper on circadian rhythms ( the term coined by Prof Franz Halberg in 1959 ) of plasma 17-OHCS in different conditions in rabbits with late Prof. K N Udupa, my mentor and teacher (8). Prof Halberg visited our world famous, the then, Surgical Research Laboratory of the Institute of Medical Sciences, Banaras Hindu University in 1977 and appreciated our work and included it for presentation during XIII International Conference of International Society for Chronobiology in Pavia (Italia) from 4-7 September 1977 and his visit to India became instrumental in formation/activation of our Indian Society for Chronobiology. In the year 2003, he came to Lucknow again on my personal invitation to inaugurate International Conference on Free Radicals and Antioxidants in Health and Disease. In the last 10 years, the cooperation between Chhatrapati Shahuji Maharaj Medical University (Formerly King George's Medical University), Lucknow and University of Minnesota, USA has been intensive and activated our participation and inclusion to the international project BIOCOS.

The international project on the BIOsphere and the COSmos originated on June 30,1997, when the Russian Academy of Medical Sciences convened a special session at its headquarters in Moscow to discuss and at the end of this meeting to unanimously endorse a project on "The BIOsphere and the COSmos" (BIOCOS). We in a worldwide project BIOCOS, are measuring blood chemistry and looking for repetitive and to that extent predictable changes that would allow time treatment known as chronotherapy. This approach by chronomics detects diagnostically the elevation of disease risk before there is actual disease. We call it preventive PREhabilitation to save the need for rehabilitation. The chronome of different biochemical variables including lipid peroxidation and anti-oxidant defense mechanisms may relate to the efficacy and management of preventive and curative chronotherapy. We have studied in detail the chronomics of different physiological variables in health and disease under tropical conditions (9-20; Fig 2a,b,c) and have demonstrated apparently for the first time, two important findings that- 


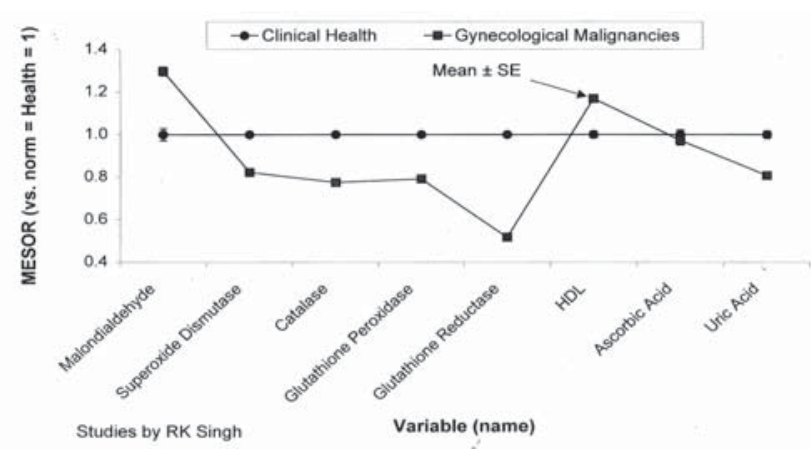

Fig 2 (a): MESOR map in gynecological malignancy vs health, showing increase of circulating malondialdehyde, one aspect desired from a putative unspecific cancer marker

1. the patients with gynecological malignancies, cirrhosis of liver, gastric ulcer and carcinoma of breast had statistically significant rhythms of studied variables, and

2. the characteristics of these rhythms were altered with statistical significance in such patients.

Chronomes of putative anti-and pro-oxidants should be mapped to explore their putative chronotherapeutic role as markers in cancer chronoprevention and management of established disease. Marker rhythm- guided individualized chronotherapy using cancer markers for the desired effect and host markers for the side effects remains in goal that has to be rendered cost effective (Fig 3). We are resuming marker rhythm guided cancer chronotherapy with kind cooperation of Prof M C Pant and Prof. M L B Bhatt in Lucknow with University of Minnesota, USA (21). Furthermore, we are studying the chronomics of 7-day/24-hour BP/HR monitoring in health and disease in our population. We measure BP/HR not only once and not only around the clock for 24-hours, but at half hourly intervals for 7-days. Moreover, we analyze not the fiction of a "true blood pressure", but the chronome of this variable as it

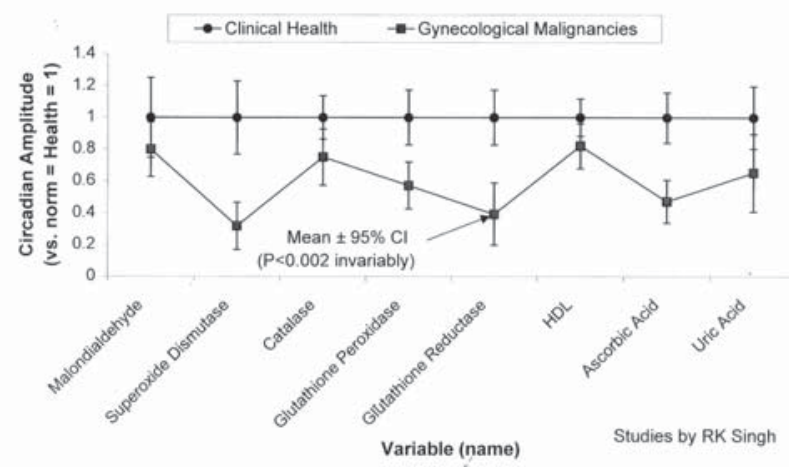

Fig 2 (b): Amplitude-map in gynecological malignancy vs health, showing non-zero circadian amplitude for malondialdehyde, an aspect desired from a putative cancer marker, also shown by other variables investigated in the human circulation

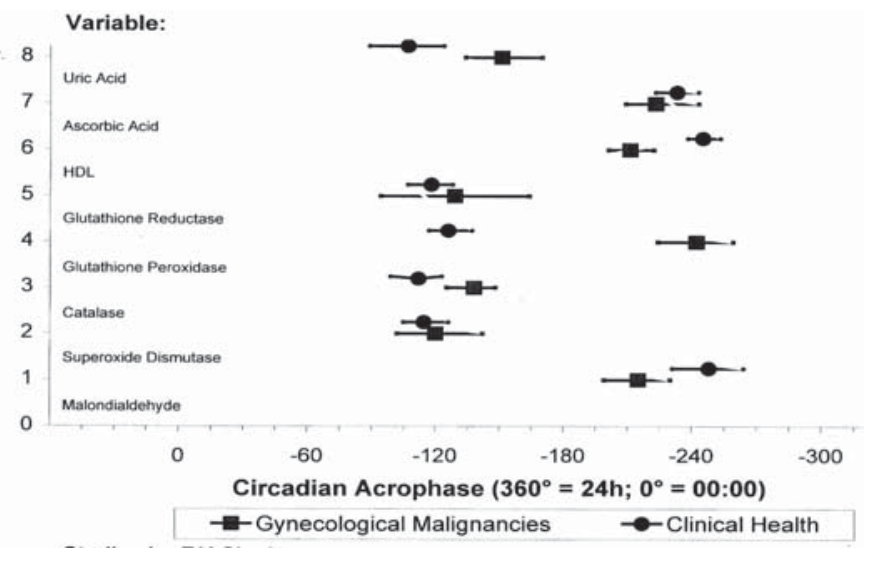

Fig 2 (c): Circadian timing of several studied variables with their $95 \%$ confidence limits in the human circulation in gynecological malignancy vs health

is much better and much cheaper to prevent stroke even when it involves an efficient chronobiologically interpreted 7-day/ 24-hour blood pressure and heart rate monitoring. Overswinging or CHAT (Circadian Hyper Amplitude Tension), that is an excessive circadian variation in blood pressure (BP), has been associated with a large increase in cardiovascular disease risk present even in the absence of an elevated BP itself. This usually asymptomatic condition is usually overlooked by current practice based on spot-checks, because to be diagnosed, measurements need to be taken around the clock, preferably for 7-days at the outset. Once diagnosed, however, a usual circadian BP pattern can be restored by means of certain non-pharmacologic or pharmacologic interventions timed appropriately. Thereby, it is possible to reduce the risk of cardiovascular morbidity and mortality, cerebral ischemic events and nephropathy in particular. A number of vascular variability disorders (VVDs) could be detected and corrected by chronobiologically analyzing ambulatory blood pressure monitoring records ( 22-24).

The role of chronomics within the context of noncommunicable diseases and mental health is mostly studied in cardiovascular variables but not limited only to the cardiovascular system. The same methodology remains applicable to a wide range of problems. Cancer prevention and optimization by the scheduling of treatment administration is another important problem. But the main focus of BIOCOS in health promotion upon the circulation by prehabilitation is to reduce the cost of rehabilitation by education of use of chronobiology (Fig 4). Thorough lifelong maps of chronomes should be generated and made available to the scientific world. Chronomic cartography from birth to death is a governmental task to implement, thereby serving the interests of 


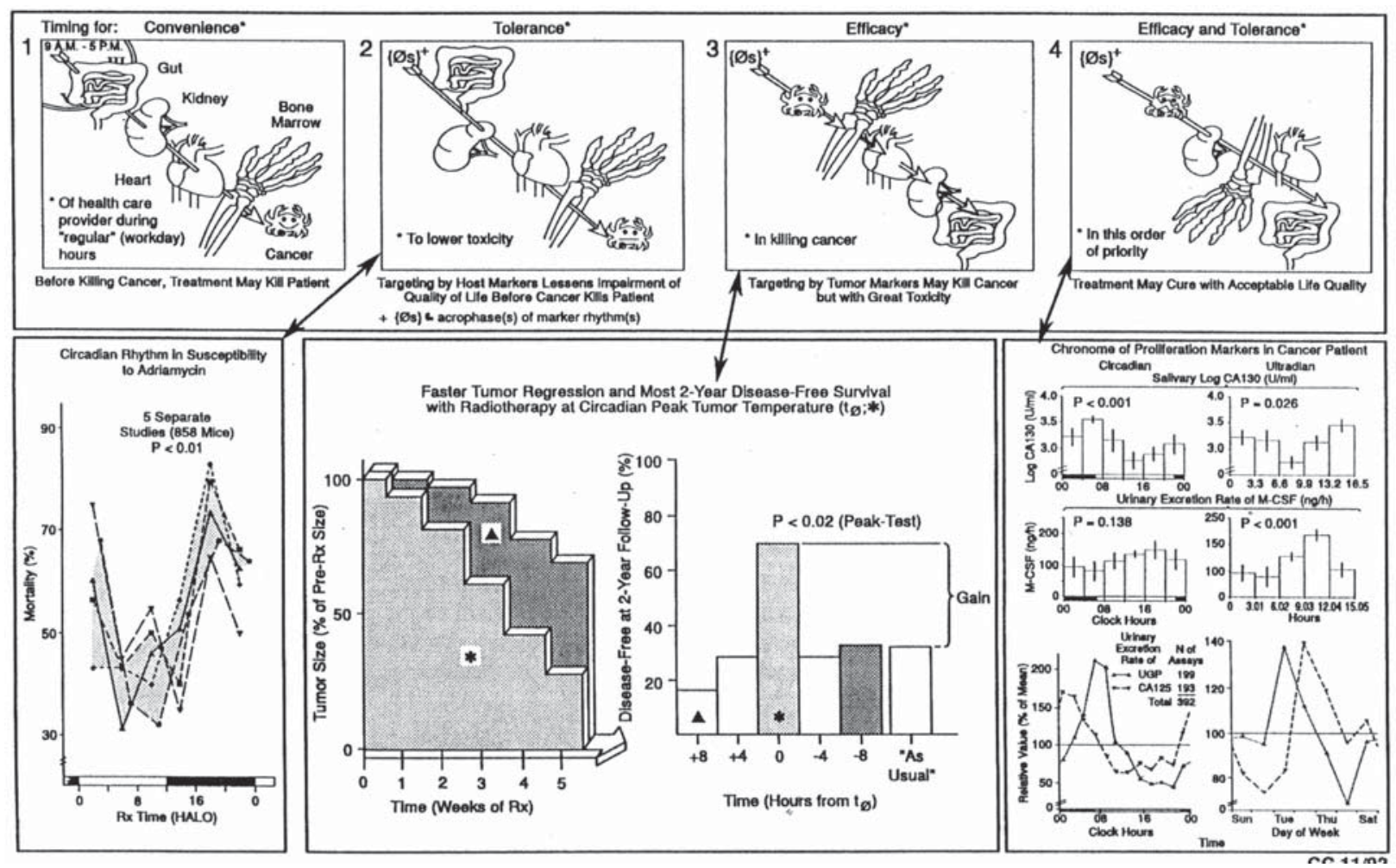

Fig 3: Principles of chronoradiotherapy (top), evolved first to lower toxicity based on the discovery of susceptibility-resistance rhythms to a variety of stimuli; eventually, we aimed for the timing of best therapeutic efficiency, goals pursued in the laboratory first, to be followed by clinical studies of chronoradiotherapy, wherein oncostatic efficiency gained major focus. The final goal is the best temporal compromise between effectiveness and tolerance, as shown abstractly at the top of this figure on the right. At the bottom on the left, toxicity studies summarize a susceptibility rhythm to adriamycin with its uncertainty; in the middle, clinical studies with timing by peak tumor temperature show faster regression and doubling of 2-year disease free survival that remains to be tested further; the promise of circadian and circaseptan cancer marker rhythms is implied at the bottom on the right. @ Halberg

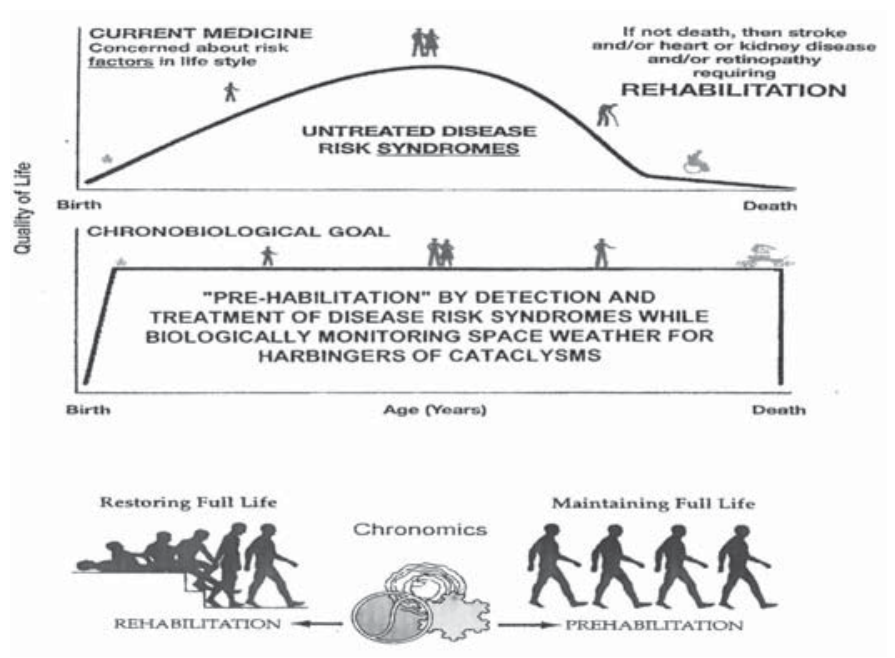

Fig 4: By the early detection of disease risk syndromes in the individual subject, countermeasures for primary prevention can be instituted. Such pre-habilitation in health can also complement rehabilitation in disease. As a major goal of health care, prehabilitation could complement an across-the-board reduction of risk factors, implemented by changes in lifestyle or drugs. (C) Halberg transdisciplinary science and general public alike for the betterment of the suffering humanity.

${ }^{*}$ Author is a member of Editorial board, IJCB and this editorial is his tribute to Father of Chronobiology: Prof. Dr. Franz Halberg.

\section{REFERENCES}

1. Halberg F. Quo vadis basic and clinical chronobiology: promise for health maintenance. Am J Anat 1983; 168: 543-94.

2. Halberg F, Halberg E. Chronopharmacology and further steps toward chronotherapy. In: Benet LZ, Massound N, Gambertoglio JG (eds): Pharmacokinetic Basis for Drug Treatment, Raven Press, New York, 1984; pp 221-48.

3. Halberg F, Cornelissen G, Bakken E. Care giving merged with chronobiologic outcome assessment, research and education in health maintenance organizations. Prog Clin Biol Res 1990; 341B: 491-549. 
4. Halberg F, Cornelissen G, Wang Z, Wan C, Ulmer W, Katinas $\mathrm{G}$, et al. Chronomics: Circadian and Circaseptan timing of radiotherapy, drugs, calories, perhaps nutriceuticals and beyond. J Exp Therapeutics Oncol 2003; 3: 223-60.

5. Halberg F, Cornelissen G, Ulmer W, Blank M, Hrushesky W, Wood P, et al. Cancer, Chronomics III. Chronomics for cancer, ageing, melatonin and experimental therapeutics researchers. J Exp Therapeutics Oncol 2006; 6: 73-84.

6. Cornelissen G, Halberg F, Schwartzkopff O, Katinas G, Johnson D, Otsuka K, et al. Editor's forward: What Gesell wished, Hellbrugge accomplished: Chronomics of child development. Neuroendocrinol Letters 2003; 24(S): 14-24.

7. Halberg F, Cornelissen G, Regal P, Otsuka K, Wang ZR, Katinas GS, et al. Chronoastrobiology: Proposal, nine conferences, heliogeomagnetics, transyears, near-weeks, near decades, phylogenetic and ontogenetic memories. Biomed Pharmacother 2004: 58: S150-S186.

8. Singh RK, Chansouria JPN, Udupa KN. Circadian periodicity of plasma cortisol (17-OHCS) levels in normal, traumatized, corticotrophin and dexamethasone treated rabbits. Ind J Med Res 1975; 63: 793-8.

9. Singh RK, Nakra VK, Pandey HN, Arora SR. Studies of circadian periodicity of plasma, breast milk and urinary calcium in lactating Indian women. Trop Geogr Med 1984; 36: 345-9.

10. Singh RK, Bansal A, Bansal SK, Rai SP. Circadian rhythms of common laboratory profiles in serum and urine of healthy Indians. Prog Clin Biol Res 1990; 341B: 559-66.

11. Singh RK, Bansal A, Bansal SK, Singh AK, Mahdi AA. Circadian periodicity of urinary inhibitor of calcium oxalate crystallization in healthy Indians and renal stone formers. Eur Urol 1993; 24: 387-92.

12. Singh R, Singh RK, Mahdi AA, Misra S, Rai SP, Singh D, et al. Studies of circadian periodicity of urinary corticoids in carcinoma of breast. In Vivo 1998; 12: 69-74.

13. Singh R, Singh RK, Mahdi AA, Saxena SP, Cornelissen G, Halberg F. Circadian periodicity of urinary volume, creatinine and 5-hydroxyindole acetic acid excretion in healthy Indians. Life Sciences 2000; 66: 209-14.

14. Singh RK, Chandra R, Narang RK, Singh SK, Katiyar SK, Singh RP, et al. Circadian variations of the absolute eosinophil count and serum histaminase activity in tropical pulmonary eosinophilia. Trop Georg Med 1987; 39: 49-52.
15. Singh RK, Singh S, Saxena S, Narang RK. Studies on circadian periodicity of plasma 17-hydroxycorticoids in tropical pulmonary eosinophilia. Prog Clin Biol Res 1987; 227B: 285-94.

16. Singh RK, Bansal A, Bansal SK. Studies on circadian periodicity of serum and urinary urate in healthy Indians and renal stone formers. Prog Clin Biol Res 1987; 227B: 305-13.

17. Singh RK, Singh Y, Srivastava R. Circadian variations of serum urea concentrations in healthy Indians. Ind J Med Res 1990; 92(B): 257-60.

18. Singh RK, Mahdi AA, Singh AK, Bansal SK, Wu J, Zhou S, et al. Circadian variation of circulating cholesterol components on vegetarian and omnivorous diets in healthy Indians. Ind J Clin Biochem 1992; 7: 185-92.

19. Singh R, Singh RK, Mahdi AA, Kumar A, Tripathi AK, Rai R, et al. Circadian periodicity of plasma lipid peroxides and antioxidants as putative markers in gynecological malignancies. In Vivo 2003; 17: 593-600.

20. Singh R, Singh RK, Tripathi AK, Cornelissen G, Schwartzkopff, Otsuka K, et al. Chronomics of circulating plasma lipid peroxides and antioxidant enzymes and other related molecules in cirrhosis of liver. Biomed Pharmacother 2005; 59: S228-S234.

21. Bhatt MLB, Singh RK, Cornelissen G, Srivastava M, Rai G, Singh $\mathrm{R}$, et al. Chronoradiotherapy guided by circadian rhythm in tumor temperature. In: Noninvasive Methods in Cardiology, Brno: Kongresove Centrum Brno1; ISBN 8086607-16-X; p. 19, 2005.

22. Germaine C, Delcourt A, Toussaint G, Otsukla K, Watanabe Y, Siegelova J, et al. Opportunity of detecting prehypertension: worldwide data on blood pressure overswinging. Biomed Pharmacother 2005; 59: S152-S156.

23. Halberg F, Cornelissen G, Otsuka K, Watanabe Y, Singh RB, Revilla M, et al. Home C- ABPM for preventive and curative health care and transdisciplinary science. World Heart $\mathrm{J}$ 2008; 1: 232-62.

24. Singh R, Verma NS, Singh RK, Singh S, Singh RB, Singh Rajesh K, et al. Continued 7-day/24-hour monitoring required in (MESOR)- Hypertension and other VVDs (Vascular Variability Disorders). World Heart J 2008; 1: 311-23. 